\title{
NEW OPERATOR INEQUALITIES IN FINITE-DIMENSIONAL VECTOR SPACES
}

\author{
ALEXANDER Y. GORDON \\ (Communicated by Michael Hitrik)
}

AbStraCt. We establish some new operator inequalities in an $n$-dimensional vector space $X$ equipped with a seminorm $\|\cdot\|$. Here is an example. If $A$ is an invertible linear operator in $X$ and $\xi$ is a vector, then

$$
\|\xi\|^{r} \leq \sum_{1 \leq|j| \leq\left(\begin{array}{c}
n+r-1 \\
r
\end{array}\right)}\left\|A^{j} \xi\right\|^{r} .
$$

Some special cases have been known and used in mathematical physics.

\section{INTRODUCTION}

Let $X$ be an $n$-dimensional real or complex vector space equipped with a seminorm $\|\cdot\|$. One of the results established in the paper states: Suppose $A_{i} \in$ $G L(X), i=1, \ldots, r$ (that is, $A_{1}, \ldots, A_{r}$ are invertible linear operators in $X$ ), and $\xi \in X$; then

$$
\|\xi\|^{r} \leq \sum_{1 \leq|j| \leq n^{r}}\left\|A_{1}^{j} \xi\right\| \ldots\left\|A_{r}^{j} \xi\right\| .
$$

In the case of identical operators, this can be improved:

$$
\|\xi\|^{r} \leq \sum_{1 \leq|j| \leq\left(\begin{array}{c}
n+r-1 \\
r
\end{array}\right)}\left\|A^{j} \xi\right\|^{r}
$$

for all $A \in G L(X)$ and all $\xi \in X$. The special cases $r=11$ and $r=2$ of this inequality (the latter case in a weaker form) have been known and used in the spectral theory of Schrödinger operators (see, e.g., [1, Theorem 10.4], [2], [3], 4], [5]).

Here is another example. For any two commuting operators $A, B \in G L(X)$ and any vector $\xi \in X$

$$
\|\xi\| \leq \sum_{1 \leq j^{2}+k^{2} \leq 4 n / \pi}\left\|A^{j} B^{k} \xi\right\| .
$$

The remaining part of the paper is organized as follows. In Section 2 we formulate and prove the main lemmas, in Section 3 we derive some consequences, and in Section 4 we apply our results to the imperfect growth model.

Received by the editors July 21, 2014.

2010 Mathematics Subject Classification. Primary 15A45, 47A63; Secondary 39A70.

${ }^{1}$ See 4 ] for its history. 


\section{LEMMAS}

Lemma 1. Suppose $g \mapsto T_{i}(g), i=1, \ldots, r$, is a linear representation of a group $G$ (with operation $\left(g_{1}, g_{2}\right) \mapsto g_{1} g_{2}$ and the identity element $e$ ) on an $n_{i}$-dimensional vector space $X_{i}$. Suppose, furthermore, that $K$ is a subset of $G$ whose cardinality $|K|$ exceeds $n_{1} \ldots n_{r}$. Then for any $r$-linear form $f$ on $X_{1} \times \ldots \times X_{r}$ and any vectors $\xi_{1} \in X_{1}, \ldots, \xi_{r} \in X_{r}$

$$
\left|f\left(\xi_{1}, \ldots, \xi_{r}\right)\right| \leq \sum_{g \in K K^{-1} \backslash\{e\}}\left|f\left(T_{1}(g) \xi_{1}, \ldots, T_{r}(g) \xi_{r}\right)\right| .
$$

Proof. We may assume that the set $K$ is finite. Given $g \in G$ and $\eta_{i} \in X_{i}(i=$ $1, \ldots, r)$, let $f_{g}\left(\eta_{1}, \ldots, \eta_{r}\right)=f\left(T_{1}(g) \eta_{1}, \ldots, T_{r}(g) \eta_{r}\right)$. The functions $f_{g}(\cdot)$ belong to the vector space $M$ of all $r$-linear forms on $X_{1} \times \ldots \times X_{r}$; the dimension of $M$ is $n_{1} \ldots n_{r}$. Since $|K|>\operatorname{dim} M$, the functions $f_{g}(\cdot), g \in K$, are linearly dependent:

$$
\sum_{g \in K} c_{g} f_{g}\left(\eta_{1}, \ldots, \eta_{r}\right)=0 \text { for all } \eta_{1} \in X_{1}, \ldots, \eta_{r} \in X_{r}
$$

not all $c_{g}$ 's being 0's. Choose $g_{0} \in K$ such that $\left|c_{g_{0}}\right|=\max _{g \in K}\left|c_{g}\right|$. Then

or

$$
f_{g_{0}}\left(\eta_{1}, \ldots, \eta_{r}\right)=\sum_{g \in K \backslash\left\{g_{0}\right\}}\left(-c_{g} / c_{g_{0}}\right) f_{g}\left(\eta_{1}, \ldots, \eta_{r}\right),
$$

$$
f\left(T_{1}\left(g_{0}\right) \eta_{1}, \ldots, T_{r}\left(g_{0}\right) \eta_{r}\right)=\sum_{g \in K \backslash\left\{g_{0}\right\}} d_{g} f\left(T_{1}(g) \eta_{1}, \ldots, T_{r}(g) \eta_{r}\right),
$$

where $\left|d_{g}\right| \leq 1$ for all $g \in K \backslash\left\{g_{0}\right\}$.

Given $\xi_{1} \in X_{1}, \ldots, \xi_{r} \in X_{r}$, let $\eta_{i}=T_{i}\left(g_{0}^{-1}\right) \xi_{i}, i=1, \ldots, r$. Then the previous equation becomes

$$
\begin{aligned}
f\left(\xi_{1}, \ldots, \xi_{r}\right) & =\sum_{g \in K \backslash\left\{g_{0}\right\}} d_{g} f\left(T_{1}\left(g g_{0}^{-1}\right) \xi_{1}, \ldots, T_{r}\left(g g_{0}^{-1}\right) \xi_{r}\right) \\
& \equiv \sum_{h \in K g_{0}^{-1} \backslash\{e\}} d_{h g_{0}} f\left(T_{1}(h) \xi_{1}, \ldots, T_{r}(h) \xi_{r}\right)
\end{aligned}
$$

so that

$$
\begin{aligned}
\left|f\left(\xi_{1}, \ldots, \xi_{r}\right)\right| & \leq \sum_{h \in K g_{0}^{-1} \backslash\{e\}}\left|f\left(T_{1}(h) \xi_{1}, \ldots, T_{r}(h) \xi_{r}\right)\right| \\
& \leq \sum_{h \in K K^{-1} \backslash\{e\}}\left|f\left(T_{1}(h) \xi_{1}, \ldots, T_{r}(h) \xi_{r}\right)\right| .
\end{aligned}
$$

In the case where the vector spaces $X_{i}$ are identical, as are representations $T_{i}(\cdot)$, and the form $f$ is symmetric, the cardinality of the set $K$ in Lemma 1 can be reduced.

Lemma 2. Suppose $g \mapsto T(g)$ is a linear representation of a group $G$ on an $n$ dimensional vector space $X$ and $f$ is a symmetric $r$-linear form on $X$. If $K$ is a subset of $G$ such that $|K|>\left(\begin{array}{c}n+r-1 \\ r\end{array}\right)$, then for any vectors $\xi_{1}, \ldots, \xi_{r} \in X$

$$
\left|f\left(\xi_{1}, \ldots, \xi_{r}\right)\right| \leq \sum_{g \in K K^{-1} \backslash\{e\}}\left|f\left(T(g) \xi_{1}, \ldots, T(g) \xi_{r}\right)\right| .
$$


The proof is similar to that of Lemma 1, the only difference is that now the functions $f_{g}\left(\eta_{1}, \ldots, \eta_{r}\right)=f\left(T(g) \eta_{1}, \ldots, T(g) \eta_{r}\right)$ belong to the vector space of symmetric $r$-linear forms on $X$ whose dimension is $\left(\begin{array}{c}n+r-1 \\ r\end{array}\right)$.

Lemma 3. Suppose $g \mapsto T_{i}(g), i=1, \ldots, r$, is a linear representation of a group $G$ on an $n_{i}$-dimensional vector space $X_{i}$ equipped with a seminorm $\|\cdot\|_{i}$. Let $K$ be a subset of $G$ such that $|K|>n_{1} \ldots n_{r}$. Then for any vectors $\xi_{1} \in X_{1}, \ldots, \xi_{r} \in X_{r}$

$$
\left\|\xi_{1}\right\|_{1} \ldots\left\|\xi_{r}\right\|_{r} \leq \sum_{g \in K K^{-1} \backslash\{e\}}\left\|T_{1}(g) \xi_{1}\right\|_{1} \ldots\left\|T_{r}(g) \xi_{r}\right\|_{r} .
$$

Proof. By the Hahn-Banach theorem, for each $i$ there is a linear functional $\varphi_{i}$ on $X_{i}$ such that $\left|\varphi_{i}\left(\eta_{i}\right)\right| \leq\left\|\eta_{i}\right\|_{i}$ for all $\eta_{i} \in X_{i}$ and $\varphi_{i}\left(\xi_{i}\right)=\left\|\xi_{i}\right\|_{i}$. Given $\eta_{1} \in$ $X_{1}, \ldots, \eta_{r} \in X_{r}$, let

$$
f\left(\eta_{1}, \ldots, \eta_{r}\right)=\varphi_{1}\left(\eta_{1}\right) \ldots \varphi_{r}\left(\eta_{r}\right)
$$

Then Lemma 1 applies, and (1) becomes

$$
\left\|\xi_{1}\right\|_{1} \ldots\left\|\xi_{r}\right\|_{r} \leq \sum_{g \in K K^{-1} \backslash\{e\}}\left|\varphi_{1}\left(T_{1}(g) \xi_{1}\right)\right| \ldots\left|\varphi_{r}\left(T_{r}(g) \xi_{r}\right)\right|,
$$

which implies (3).

The following statement is the version of Lemma 3 in the case where the vector spaces $X_{1}, \ldots, X_{r}$ are identical, the representations $g \mapsto T_{i}(g)$ are identical as well, and $\xi_{1}=\ldots=\xi_{r}$.

Lemma 4. Suppose $g \mapsto T(g)$ is a linear representation of a group $G$ on an $n$ dimensional vector space $X$ equipped with a seminorm $\|\cdot\|$. Let $K$ be a subset of $G$ such that $|K|>\left(\begin{array}{c}n+r-1 \\ r\end{array}\right)$. Then for any vector $\xi \in X$

$$
\|\xi\|^{r} \leq \sum_{g \in K K^{-1} \backslash\{e\}}\|T(g) \xi\|^{r} .
$$

Proof. For $\eta_{1}, \ldots, \eta_{r} \in X$, let $f\left(\eta_{1}, \ldots, \eta_{r}\right)=\varphi\left(\eta_{1}\right) \ldots \varphi\left(\eta_{r}\right)$, where $\varphi$ is a linear functional on $X$ such that $\varphi(\xi)=\|\xi\|$ and $|\varphi(\eta)| \leq\|\eta\|$ for all $\eta \in X$. Since $f$ is a symmetric $r$-linear form on $X$, Lemma 2 is applicable, and (2) gives

$$
\begin{aligned}
\|\xi\|^{r}=f(\xi, \ldots, \xi) & \leq \sum_{g \in K K^{-1} \backslash\{e\}}|\varphi(T(g) \xi)| \ldots|\varphi(T(g) \xi)| \\
& \leq \sum_{g \in K K^{-1} \backslash\{e\}}\|T(g) \xi\|^{r} .
\end{aligned}
$$

\section{TheOrems}

In this section, $\|\cdot\|$ is an arbitrary seminorm in an $n$-dimensional vector space $X$, and $\|\cdot\|_{i}$ is any seminorm in an $n_{i}$-dimensional vector space $X_{i}(i=1, \ldots, r)$.

Theorem 1. Let $\xi_{i} \in X_{i}$ and $A_{i} \in G L\left(X_{i}\right)(i=1, \ldots, r)$. Then

$$
\left\|\xi_{1}\right\|_{1} \ldots\left\|\xi_{r}\right\|_{r} \leq \sum_{1 \leq|j| \leq n_{1} \ldots n_{r}}\left\|A_{1}^{j} \xi_{1}\right\|_{1} \ldots\left\|A_{r}^{j} \xi_{r}\right\|_{r} .
$$

Proof. Apply Lemma 3 with $G=\mathbf{Z}, K=\left\{j \in \mathbf{Z} \mid 0 \leq j \leq n_{1} \ldots n_{r}\right\}$ and $T_{i}(j)=$ $A_{i}^{j}(1 \leq i \leq r ; j \in \mathbf{Z})$. 
Theorem 2. If $\xi \in X$ and $A \in G L(X)$, then

$$
\|\xi\|^{r} \leq \sum_{1 \leq|j| \leq\left(\begin{array}{c}
n+r-1 \\
r
\end{array}\right)}\left\|A^{j} \xi\right\|^{r} .
$$

Proof. Apply Lemma 4 with $G=\mathbf{Z}, K=\left\{j \in \mathbf{Z} \mid 0 \leq j \leq\left(\begin{array}{c}n+r-1 \\ r\end{array}\right)\right\}$ and $T(j)=$ $A^{j}(j \in \mathbf{Z})$.

Example 1. Suppose for each $i=1, \ldots, r$ the function $y_{i}(\cdot)$ is a solution of the differential equation

$$
y_{i}^{\left(p_{i}\right)}(t)+\sum_{m=0}^{p_{i}-1} a_{i, m}(t) y_{i}^{(m)}(t)=0, \quad-\infty<t<\infty,
$$

whose coefficients $a_{i, m}(\cdot)$ are $C^{\infty}$-smooth and $T$-periodic. Then for any integers $k_{i} \geq 0$ and any $a \in \mathbf{R}$

$$
\left|y_{1}^{\left(k_{1}\right)}(a)\right| \ldots\left|y_{r}^{\left(k_{r}\right)}(a)\right| \leq \sum_{1 \leq|j| \leq p_{1} \ldots p_{r}}\left|y_{1}^{\left(k_{1}\right)}(a+j T)\right| \ldots\left|y_{r}^{\left(k_{r}\right)}(a+j T)\right| .
$$

Proof. The vector space $X_{i}$ of all solutions of (6) is $p_{i}$-dimensional. The operator $A_{i}: z(\cdot) \rightarrow z(\cdot+T)$ maps $X_{i}$ onto itself. It remains to apply Theorem 11 to these operators, the seminorms $\|z\|_{i}=\left|z^{\left(k_{i}\right)}(a)\right|\left(z \in X_{i}\right)$ and the vectors $y_{i} \in X_{i}, i=$ $1, \ldots, r$.

Let, in particular, $y(\cdot)$ be a solution of the differential equation

$$
y^{\prime \prime}(t)+b(t) y^{\prime}(t)+c(t) y(t)=0, \quad t \in \mathbf{R},
$$

with $T$-periodic $\mathbf{C}^{\infty}$-smooth complex-valued coefficients $b(\cdot)$ and $c(\cdot)$. Then for any $a \in \mathbf{R}$ and any $r \in \mathbf{N}, k \in \mathbf{Z}_{+}$

$$
\left|y^{(k)}(a)\right|^{r} \leq \sum_{1 \leq|j| \leq r+1}\left|y^{(k)}(a+j T)\right|^{r}
$$

(here we use Theorem 2 rather than Theorem 1).

Example 2. Let $P(\cdot)$ be a polynomial of degree $m$. Then for any $z, h \in \mathbf{C} \backslash\{0\}$

$$
|P(z)| \leq \sum_{1 \leq|j| \leq m+1}\left|P\left(h^{j} z\right)\right| .
$$

The following statement generalizes this example.

Corollary 1. Let $P(\zeta) \equiv P\left(\zeta_{1}, \ldots, \zeta_{v}\right)$ be a polynomial in $v$ variables of degree $d$. Let $B$ be an invertible affine transformation of $\mathbf{C}^{v}$. Then for any $\zeta \in \mathbf{C}^{v}$

$$
|P(\zeta)| \leq \sum_{1 \leq|j| \leq\left(\begin{array}{c}
d+v \\
v
\end{array}\right)}\left|P\left(B^{j} \zeta\right)\right|
$$

Proof. We note that the vector space of all such polynomials has dimension $\left(\begin{array}{c}d+v \\ v\end{array}\right)$; then we apply Theorem 2 with $r=1$.

Theorem 3. Let $\xi_{i} \in X_{i}$ and $A_{i, k} \in G L\left(X_{i}\right)(i=1, \ldots, r ; k=1, \ldots, s)$. Suppose the operators $A_{i, k}$ with any fixed $i$ commute. If $K$ is a subset of $\mathbf{Z}^{s}$ such that $|K|>n_{1} \ldots n_{r}$, then

$$
\left\|\xi_{1}\right\|_{1} \ldots\left\|\xi_{r}\right\|_{r} \leq \sum_{\mathbf{j} \in(K-K) \backslash\{0\}}\left\|A_{1,1}^{j_{1}} \ldots A_{1, s}^{j_{s}} \xi_{1}\right\|_{1} \ldots\left\|A_{r, 1}^{j_{1}} \ldots A_{r, s}^{j_{s}} \xi_{r}\right\|_{r} .
$$


Proof. Apply Lemma 3 with $G=\mathbf{Z}^{s}$ and, for any $\mathbf{j}=\left(j_{1}, \ldots, j_{s}\right) \in \mathbf{Z}^{s}, T_{i}(\mathbf{j})=$ $A_{i, 1}^{j_{1}} \ldots A_{i, s}^{j_{s}}$.

Theorem 4. Suppose operators $A_{1}, \ldots, A_{s} \in G L(X)$ commute. If $K$ is a subset of $\mathbf{Z}^{s}$ such that $|K|>\left(\begin{array}{c}n+r-1 \\ r\end{array}\right)$, then for any vector $\xi \in X$

$$
\|\xi\|^{r} \leq \sum_{\mathbf{j} \in(K-K) \backslash\{0\}}\left\|A_{1}^{j_{1}} \ldots A_{s}^{j_{s}} \xi\right\|^{r} .
$$

Proof. Apply Lemma 4 with $G=\mathbf{Z}^{s}$ and $T(\mathbf{j})=A_{1}^{j_{1}} \ldots A_{s}^{j_{s}}$ for all $\mathbf{j}=\left(j_{1}, \ldots, j_{s}\right) \in$ $\mathbf{Z}^{s}$.

Theorem 5. Let $H$ be a closed convex centrally symmetric set in $\mathbf{R}^{s}$. If operators $A_{1}, \ldots, A_{s}$ commute and $\operatorname{Vol}(H) \geq 2^{s}\left(\begin{array}{c}n+r-1 \\ r\end{array}\right)$, then for any vector $\xi \in X$

$$
\|\xi\|^{r} \leq \sum_{\mathbf{j} \in\left(H \cap \mathbf{Z}^{s}\right) \backslash\{0\}}\left\|A_{1}^{j_{1}} \ldots A_{s}^{j_{s}} \xi\right\|^{r} .
$$

Proof. We may assume that $H$ is bounded and hence $\operatorname{Vol}(H)<\infty$. Let $G=\frac{1}{2} H$. There is $x_{0} \in \mathbf{R}^{s}$ such that $\left|\left(G-x_{0}\right) \cap \mathbf{Z}^{s}\right|>\operatorname{Vol}(G)$. Indeed, by shifting $G$ we may assume that $G$ has a boundary point, say $a$, in the interior of the cube $Q=\left\{x \in \mathbf{R}^{s}: 0 \leq x_{i}<1, i=1, \ldots, s\right\}$. Let $G_{\mathbf{j}}=(G-\mathbf{j}) \cap Q\left(\mathbf{j} \in \mathbf{Z}^{s}\right)$ and $f(x)=\sum_{\mathbf{j} \in \mathbf{Z}^{s}} \mathbf{1}_{G_{\mathbf{j}}}(x)(x \in Q)$. Then

$$
\int_{Q} f(x) d x=\sum_{\mathbf{j} \in \mathbf{Z}^{s}} \int_{Q} \mathbf{1}_{G_{\mathbf{j}}}(x) d x=\sum_{\mathbf{j} \in \mathbf{Z}^{s}} \operatorname{Vol}\left(G_{\mathbf{j}}\right)=\operatorname{Vol}(G) .
$$

There is $x_{0} \in Q$ such that $f\left(x_{0}\right)>\operatorname{Vol}(G)$. (Assume the converse; then, in particular, $f(a) \leq \operatorname{Vol}(G)$. Consequently, we have $f(x)=f(a)-1 \leq \operatorname{Vol}(G)-1$ for all points $x \in Q \backslash G$ that are close enough to $a$, hence on a nonempty open subset of $Q$; together with our assumption, this implies that $\int_{Q} f(x) d x<\operatorname{Vol}(G)$, which contradicts (9).) It remains to note that $f\left(x_{0}\right)=\left|\left\{\mathbf{j} \in \mathbf{Z}^{s}: x_{0} \in G-\mathbf{j}\right\}\right|=\mid\{\mathbf{j} \in$ $\left.\mathbf{Z}^{s}: \mathbf{j} \in G-x_{0}\right\}|=|\left(G-x_{0}\right) \cap \mathbf{Z}^{s} \mid$.

Let $K=\left(G-x_{0}\right) \cap \mathbf{Z}^{s}$. Then $K-K \subset(G-G) \cap \mathbf{Z}^{s}=H \cap \mathbf{Z}^{s}$. Since $|K|>$ $\operatorname{Vol}(G)=2^{-s} \operatorname{Vol}(H) \geq\left(\begin{array}{c}n+r-1 \\ r\end{array}\right)$, the inequality (8) follows from Theorem 4.

Example 3. Let $A, B \in G L(X)$ be two commuting operators. Let $r \in \mathbf{N}$ and $H=\left\{(x, y) \in \mathbf{R}^{2}: x^{2}+y^{2} \leq 4\left(\begin{array}{c}n+r-1 \\ r\end{array}\right) / \pi\right\}$. The area of the disc $H$ equals $4\left(\begin{array}{c}n+r-1 \\ r\end{array}\right)$ and, according to Theorem 5 with $s=2$, for any vector $\xi \in X$

$$
\|\xi\|^{r} \leq \sum_{1 \leq j^{2}+k^{2} \leq 4\left(\begin{array}{c}
n+r-1 \\
r
\end{array}\right) / \pi}\left\|A^{j} B^{k} \xi\right\|^{r} .
$$

Consider, as a special case, the equation

$$
(\Delta+v) y=\lambda y
$$

on the "discrete torus" $\mathbf{Z}_{L}^{2}=(\mathbf{Z} / L \mathbf{Z})^{2}$. Here $(\Delta y)\left(x_{1}, x_{2}\right)=y\left(x_{1}+1, x_{2}\right)+$ $y\left(x_{1}-1, x_{2}\right)+y\left(x_{1}, x_{2}+1\right)+y\left(x_{1}, x_{2}-1\right)$ and $(v y)(x)=v(x) y(x)$, where $v(\cdot)$ is a $(\tau, \tau)$-periodic function on $\mathbf{Z}_{L}^{2}, \tau$ being a divisor of $L$. If $y(\cdot)$ is a solution of (11), then for any $x=\left(x_{1}, x_{2}\right) \in \mathbf{Z}_{L}^{2}$

$$
\left|y\left(x_{1}, x_{2}\right)\right| \leq \sum_{1 \leq j^{2}+k^{2} \leq 8 L / \pi}\left|y\left(x_{1}+j \tau, x_{2}+k \tau\right)\right| .
$$


Indeed, consider the space $X$ of all solutions of (11); its dimension is $\leq 2 L$. Also consider the operators $A$ and $B$ acting in $X$ as follows:

$$
(A y)\left(x_{1}, x_{2}\right)=y\left(x_{1}+\tau, x_{2}\right) \text { and }(B y)\left(x_{1}, x_{2}\right)=y\left(x_{1}, x_{2}+\tau\right) .
$$

They commute; it remains to apply (10) with $r=1$. Note that the inequality (12) is nontrivial only if $\tau^{2}<\pi L / 8$ (otherwise, the right-hand side contains terms identical to the one on the left).

\section{An APPLICATION}

In this section we show how inequalities of the type considered in the present work can be used in spectral theory. Theorem [6 below is a stronger version of the main result (Theorem 1 ) of 4 .

We consider a discrete model of the $d$-dimensional medium whose Hamiltonian is the Schrödinger operator $h_{v}$ acting in $l^{2}\left(\mathbf{Z}^{d}\right): h_{v}=\Delta+v$, where $\Delta$ is the lattice Laplacian and $v$ is the operator of multiplication by the lattice potential $v(\cdot)$. In this model, the potential $v(\cdot)$ is the result of the following recursive process. Consider a nested sequence of discrete cubes $Q_{n}(n=0,1,2, \ldots)$ of the form

$$
Q_{n}=\left\{x \in \mathbf{Z}^{d}: \quad-\frac{T_{n}}{2}<x_{i} \leq \frac{T_{n}}{2}, \quad i=1, \ldots, d\right\}
$$

whose sides $T_{n} \in \mathbf{N}$ are such that

$$
\frac{T_{n}}{T_{n-1}} \in \mathbf{N} \backslash\{1\}, \quad n=1,2,3, \ldots
$$

Initially, the potential is defined on the cube $Q_{0}$. At the $n$th step $(n=1,2, \ldots)$ the potential, already defined on the cube $Q_{n-1}$, is extended $Q_{n-1}$-periodically to the cube $Q_{n}$; then "impurities" are being introduced by arbitrarily changing the values of the potential at $m_{n}$ points of $Q_{n}$ selected at random so that all such selections are equiprobable. After infinitely many steps, we obtain a potential $v(\cdot)$ on $\mathbf{Z}^{d}$, which is almost surely (a.s.) well-defined if

$$
\sum_{n=1}^{\infty} m_{n} / T_{n}^{d}<\infty
$$

(due to the Borel-Cantelli Lemma).

We are going to show that if the sequence $T_{n}$ grows fast enough while the sequence $m_{n}$ grows not too fast, then a.s. the operator $h_{v}$ does not have eigenvaluesin other words, there are no localized states 2 Before formulating the result precisely, we will describe our model in an equivalent but more convenient way.

We consider a Schrödinger operator $h_{v}=\Delta+v$ acting in $l^{2}\left(\mathbf{Z}^{d}\right)$ as follows:

$$
\left(h_{v} y\right)(x)=\sum_{z \in \mathbf{Z}^{d}:\|z\|_{1}=1} y(x+z)+v(x) y(x), \quad x \in \mathbf{Z}^{d},
$$

where $\|z\|_{1}=\left|z_{1}\right|+\ldots+\left|z_{d}\right|$ and

$$
v(x)=\sum_{n=0}^{\infty} u_{n}(x) .
$$

\footnotetext{
${ }^{2}$ Note that if $m_{n}=0$ for $n \geq n_{0}$, then the potential $v$ is periodic, in which case the spectrum of $h_{v}$ is well known to be not only continuous (no eigenvalues) but absolutely continuous.
} 
Here $u_{n}(\cdot)$ is a $\left(T_{n}, \ldots, T_{n}\right)$-periodic function on $\mathbf{Z}^{d}$, where the numbers $T_{n} \in$ $\mathbf{N}(n=0,1,2, \ldots)$ satisfy the condition (14). We assume that the function $u_{n}(\cdot)$ $(n \geq 1)$ on its elementary cell $Q_{n}$ defined by (13) vanishes everywhere except for a uniformly distributed random subset $X_{n} \subset Q_{n}$ of cardinality $m_{n}$. The values of $u_{n}(\cdot)$ on $X_{n}$ are arbitrary. Under the condition (15) the potential $v$ is a.s. well-defined, and $h_{v}$ is a linear operator in $l^{2}\left(\mathbf{Z}^{d}\right)$ (in general, unbounded) with the domain $D\left(h_{v}\right)=\left\{y \in l^{2}\left(\mathbf{Z}^{d}\right): \quad \sum_{x \in \mathbf{Z}^{d}}|v(x) y(x)|^{2}<\infty\right\}$. In our model, the functions $u_{n}$ are real-valued so the operator $h_{v}$ is self-adjoint. However, in what follows the functions $u_{n}$ may be complex-valued.

Theorem 6. If

$$
\sum_{n=0}^{\infty} \frac{m_{n+1} T_{n}^{2 d-1}}{T_{n+1}}<\infty,
$$

then with probability 1 the operator (16), (17) has no eigenvalues.

The proof of Theorem 6 is based on a theorem regarding solutions of periodic lattice equations, which is an improvement of the corresponding statement (Theorem 2 ) in [4]. For a function $y: \mathbf{Z}^{d} \rightarrow \mathbf{C}$, let

$$
\sigma(k)=\sum_{x \in \mathbf{Z}^{d}: x_{d}=k}|y(x)|^{2}, \quad k \in \mathbf{Z} \quad(0 \leq \sigma(k) \leq \infty) .
$$

Theorem 7. Let $y: \mathbf{Z}^{d} \rightarrow \mathbf{C}$ be a solution of $(\Delta+q) y=\lambda y$, where $q(\cdot)$ is a $\left(\tau_{1}, \ldots, \tau_{d}\right)$-periodic complex-valued function on $\mathbf{Z}^{d}$. Suppose $\sigma(k)<\infty$ for all $k \in \mathbf{Z}$. Then

$$
\sigma(0) \leq \sum_{1 \leq|m| \leq 2 N^{2}+N} \sigma\left(m \tau_{d}\right)
$$

where $N=\tau_{1} \ldots \tau_{d-1}$.

In order to simplify the notation, we will present the proofs of Theorems 6 and 7 only for $d=2$. The proofs in the general case $(d \geq 2)$ follow the same lines.

Proof of Theorem $7(d=2)$. The periods $\tau_{1}$ and $\tau_{2}$ of the function $q$ will be alternatively denoted as $J$ and $K$, and the coordinates of a generic point $x \in \mathbf{Z}^{2}$ as $j$ and $k$. We want to show that if $y(\cdot)$ satisfies the equation $h_{q} y=\lambda y$ and

$$
\sigma(k) \equiv \sum_{j \in \mathbf{Z}}|y(j, k)|^{2}<\infty \quad \text { for all } k \in \mathbf{Z}
$$

then

$$
\sigma(0) \leq \sum_{1 \leq|m| \leq 2 J^{2}+J} \sigma(m K) .
$$

(a) Let $y: \mathbf{Z}^{2} \rightarrow \mathbf{C}$ be a solution of the equation $h_{q} y=\lambda y$, i.e., of the equation (21)

$y(j, k+1)+y(j, k-1)+y(j+1, k)+y(j-1, k)+(q(j, k)-\lambda) y(j, k)=0 \quad(j, k \in \mathbf{Z})$, satisfying (19). Consider the "discrete cylinder" $C_{J}=\mathbf{Z}_{J} \times \mathbf{Z}$, where $\mathbf{Z}_{J}=\mathbf{Z} / J \mathbf{Z}$, and the function $Z(\nu, k ; \theta)$ on $C_{J} \times \mathbf{T}$ (where $\mathbf{T}=\mathbf{R} / \mathbf{Z}$ ), setting

$$
Z(\nu, k ; \theta)=\sum_{j \in \nu} y(j, k) e^{2 \pi i j \theta} .
$$


In view of (19), for any fixed $\nu \in \mathbf{Z}_{J}$ and $k \in K$, this series converges in $L^{2}(\mathbf{T})$ and, by Parseval's identity,

$$
\int_{\mathbf{T}}|Z(\nu, k ; \theta)|^{2} d \theta=\sum_{j \in \nu}|y(j, k)|^{2} .
$$

The $(J, K)$-periodic function $q(j, k)$ carries over naturally to $C_{J}$, generating a function $Q(\nu, k)\left(\nu \in \mathbf{Z}_{J}, k \in \mathbf{Z}\right)$, which is $K$-periodic in $k$. It is easy to derive from (21) that for any $\nu \in \mathbf{Z}_{J}$ and $k \in K$ one has the following equality in $L^{2}(\mathbf{T})$ :

$$
\begin{aligned}
Z(\nu, k+1 ; \theta)+Z(\nu, k-1 ; \theta) & +e^{-2 \pi i \theta} Z(\nu+1, k ; \theta) \\
& +e^{2 \pi i \theta} Z(\nu-1, k ; \theta)+(Q(\nu, k)-\lambda) Z(\nu, k ; \theta)=0 .
\end{aligned}
$$

Each $Z(\nu, k ; \cdot)$, as an element of $L^{2}(\mathbf{T})$, is a class of equivalent square integrable Lebesgue measurable functions on $\mathbf{T}$. Let us select a Borel measurable function in this class. From now on, $Z(\nu, k ; \cdot)$ will denote that function on $\mathbf{T}$, and each equation (23) will be understood as a pointwise equation that holds for Lebesgue a.e. $\theta \in \mathbf{T}$. Let $B$ be a full measure Borel subset of $\mathbf{T}$ such that (23) holds for any $\theta \in B$ and all $\nu \in \mathbf{Z}_{J}, k \in \mathbf{Z}$.

(b) Denote by $\mathcal{M}$ the $J$-dimensional coordinate vector space over $\mathbf{C}$ with coordinates labeled by elements of $\mathbf{Z}_{J}$. Pick an arbitrary $\theta \in B$. Setting $\zeta_{k}(\theta)=$

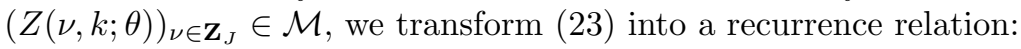

$$
\zeta_{k+1}(\theta)=L_{k}(\theta) \zeta_{k}(\theta)-\zeta_{k-1}(\theta),
$$

where $L_{k}(\theta)$ is the linear operator in $\mathcal{M}$ acting on vectors $(\zeta(\nu))_{\nu \in \mathbf{Z}_{J}} \in \mathcal{M}$ as follows:

$$
\left(L_{k}(\theta) \zeta\right)(\nu)=-e^{2 \pi i \theta} \zeta(\nu-1)-e^{-2 \pi i \theta} \zeta(\nu+1)-V(\nu, k) \zeta(\nu) .
$$

Since $V(\nu, k)$ is $K$-periodic in $k$, we have $L_{k+K}(\theta)=L_{k}(\theta)$ for any $k \in \mathbf{Z}$.

Let $\mathcal{L}=\mathcal{M} \oplus \mathcal{M}$ and $\xi_{k}(\theta)=\left(\zeta_{k}(\theta), \zeta_{k-1}(\theta)\right) \in \mathcal{L}$. The equation (24) can be further transformed into

$$
\xi_{k+1}(\theta)=T_{k}(\theta) \xi_{k}(\theta),
$$

where the operator $T_{k}(\theta) \in G L(\mathcal{L})$ acts as follows: given $\zeta, \eta \in \mathcal{M}$,

$$
T_{k}(\theta)((\zeta, \eta))=\left(-\eta+L_{k}(\theta) \zeta, \zeta\right) .
$$

Again, $T_{k}(\theta)$ is $K$-periodic in $k$ :

$$
T_{k+K}(\theta)=T_{k}(\theta) .
$$

Let, furthermore,

$$
S(\theta)=T_{K-1}(\theta) T_{K-2}(\theta) \ldots T_{1}(\theta) T_{0}(\theta) .
$$

In view of (25) and (26),

$$
\xi_{r K}(\theta)=(S(\theta))^{r} \xi_{0}(\theta)
$$

for all $r \in \mathbf{Z}$.

(c) By (27) and Theorem 2 with $r=2$, for any seminorm $\|\cdot\|$ in $\mathcal{L}$

$$
\left\|\xi_{0}(\theta)\right\|^{2} \leq \sum_{1 \leq|m| \leq\left(\begin{array}{c}
2 J+1 \\
2
\end{array}\right)}\left\|\xi_{m K}(\theta)\right\|^{2} .
$$


Define a seminorm $\|\cdot\|$ in $\mathcal{L}$ as follows. Let $\xi \in \mathcal{L}$, that is, $\xi=(\zeta, \eta)$, where $\zeta, \eta \in \mathcal{M}$. Then we set $\|\xi\|=\left(\sum_{\nu \in \mathbf{Z}_{J}}\left|\zeta_{\nu}\right|^{2}\right)^{1 / 2}$. Now the inequality (28) implies that

$$
\sum_{\nu \in \mathbf{Z}_{J}}|Z(\nu, 0 ; \theta)|^{2} \leq \sum_{1 \leq|m| \leq 2 J^{2}+J}\left(\sum_{\nu \in \mathbf{Z}_{J}}|Z(\nu, m K ; \theta)|^{2}\right),
$$

which is true for all $\theta \in B$. Integrating both sides of this inequality over $B$ or, equivalently, over $\mathbf{T}$ and using (22), we derive that

$$
\sum_{j \in \mathbf{Z}}|y(j, 0)|^{2} \leq \sum_{1 \leq|m| \leq 2 J^{2}+J}\left(\sum_{j \in \mathbf{Z}}|y(j, m K)|^{2}\right),
$$

which is equivalent to (20).

Proof of Theorem 6 $(d=2)$. For all $n \geq 0$ and $x \in \mathbf{Z}^{2}$, let $v_{n}(x)=u_{0}(x)+\ldots+$ $u_{n}(x)$. Consider the elementary cell $Q_{n+1}$ of $v_{n+1}(\cdot)$ :

$$
Q_{n+1}=\left\{x=(j, k) \in \mathbf{Z}^{2}:-T_{n+1} / 2<j, k \leq T_{n+1} / 2\right\}
$$

and the $\operatorname{strip} S_{n}=\left\{x=(j, k) \in \mathbf{Z}^{2}: \quad-W_{n} \leq k \leq W_{n}\right\}$, where $W_{n}=2 T_{n}^{3}+T_{n}^{2}$. Let

$$
p_{n+1}=\operatorname{Pr}\left\{X_{n+1} \cap S_{n} \neq \emptyset\right\} \equiv \operatorname{Pr}\left\{X_{n+1} \cap R_{n+1} \neq \emptyset\right\},
$$

where $R_{n+1}=Q_{n+1} \cap S_{n}$. It is easy to see that

$$
p_{n+1} \leq m_{n+1} \frac{2 W_{n}+1}{T_{n+1}} .
$$

By the Borel-Cantelli Lemma, the inequality $\sum_{n>0} p_{n}<\infty$, which follows from (18), implies that a.s. there is a random number $N(\bar{\omega})$ such that $X_{n+1} \cap S_{n}=\emptyset$ for all $n \geq N(\omega)$. Consequently, for all $n \geq N(\omega)$ we have $\left(X_{n+1} \cup X_{n+2} \cup \ldots\right) \cap S_{n}=\emptyset$ so that on the $\operatorname{strip} S_{n} u_{n+1}(x)=u_{n+2}(x)=\ldots=0$ and, therefore,

$$
v(x)=v_{n}(x) \quad \text { on } S_{n} \text { for } n \geq N(\omega) .
$$

Suppose $y(\cdot)$ is a solution of $(\Delta+v) y=\lambda y$ that belongs to $l^{2}\left(\mathbf{Z}^{2}\right)$, so that $\sum_{k \in \mathbf{Z}} \sigma(k)<\infty$. For any $n \geq 0$, denote by $y_{n}(\cdot)$ the unique function on $\mathbf{Z}^{2}$ satisfying the equation $\left(\Delta+v_{n}\right) y_{n}=\lambda y_{n}$ and such that $y_{n}(j, k)=y(j, k)$ for $k=-1,0$ and all $j \in \mathbf{Z}$. Let $\sigma_{n}(k)=\sum_{j \in \mathbf{Z}}\left|y_{n}(j, k)\right|^{2}, k \in \mathbf{Z}$.

It follows from Theorem 7 (with $\tau_{1}=\tau_{2}=T_{n}$ ) that

$$
\sigma_{n}(0) \leq \sum_{1 \leq|m| \leq 2 T_{n}^{2}+T_{n}} \sigma_{n}\left(m T_{n}\right) \leq \sum_{T_{n} \leq|k| \leq W_{n}} \sigma_{n}(k) .
$$

Due to (29), we have $y_{n}(x)=y(x)$ on the strip $S_{n}$ if $n \geq N(\omega)$, and hence

$$
\sigma(0) \leq \sum_{T_{n} \leq|k| \leq W_{n}} \sigma(k)
$$

for $n \geq N(\omega)$. Therefore, a.s. $\sigma(0) \leq \sum_{|k| \geq T_{n}} \sigma(k)$ for all large enough $n$; since $\sum_{k \in \mathbf{Z}} \sigma(k)<\infty$ and $T_{n} \rightarrow \infty$ as $n \rightarrow \infty$, it follows that $\sigma(0)=0$, that is, $y(j, 0)=0$ for all $j \in \mathbf{Z}$.

Similarly, the function $y^{*}(j, k)=y(j, k+1)$ satisfies the equation $\left(\Delta+v^{*}\right) y^{*}=$ $\lambda y^{*}$, where $v^{*}(j, k)=v(j, k+1)$ for all $j, k$. The potential $v^{*}(\cdot)$ has the same structure as $v(\cdot)$ (since in the construction of $v(\cdot)$ each square $Q_{n}$ could be replaced 
by any shift thereof), so the inclusion $y^{*} \in l^{2}\left(\mathbf{Z}^{2}\right)$ a.s. implies that $y^{*}(j, 0)=0$ for all $j$.

Therefore, if $(\Delta+v) y=\lambda y$ and $y \in l^{2}\left(\mathbf{Z}^{2}\right)$, then a.s. $y(j, 0)=y(j, 1)=0$ for all $j \in \mathbf{Z}$ and hence $y \equiv 0$ on $\mathbf{Z}^{2}$.

\section{ACKNOWLEDGMENT}

The author is grateful to the anonymous referee for the careful reading of the manuscript and helpful comments and suggestions.

\section{REFERENCES}

[1] H. L. Cycon, R. G. Froese, W. Kirsch, B. Simon. Schrödinger Operators with Application to Quantum Mechanics and Global Geometry. Springer, corrected edition: Berlin, 2007.

[2] David Damanik, Gordon-type arguments in the spectral theory of one-dimensional quasicrystals, Directions in mathematical quasicrystals, CRM Monogr. Ser., vol. 13, Amer. Math. Soc., Providence, RI, 2000, pp. 277-305. MR1798997 (2002c:81048)

[3] A. Ya. Gordon, A sufficient condition for continuity of the spectrum of a discrete Schrödinger operator (Russian), Funktsional. Anal. i Prilozhen. 20 (1986), no. 4, 70-71. MR878048 (88d:39011)

[4] A. Gordon, Imperfectly grown periodic medium: absence of localized states, J. Spectr. Theory 5 (2015), no. 2, 279-294, DOI 10.4171/JST/98. MR3355452

[5] S. Jitomirskaya, Wen-Cai Liu. Arithmetic spectral transitions for the Maryland model, Preprint, 2014.

Department of Mathematics and Statistics, University of North Carolina at Charlotte, 9201 University City Blvd, Charlotte, North Carolina 28223

E-mail address: aygordon@uncc.edu 